\title{
Dentate nucleus connectivity in adult patients with multiple sclerosis: functional changes at rest and correlation with clinical features
}

\author{
Emilia Sbardella, Neeraj Upadhyay, Francesca Tona, Luca Prosperini, Laura De Giglio, \\ Nikolaos Petsas, Carlo Pozzilli and Patrizia Pantano
}

\section{Abstract}

Background and objective: The dentate nucleus, which is the largest of the cerebellar nuclei, plays a critical role in movement and cognition. The aim of our study was to assess any changes in dentate functional connectivity (FC) in adult relapsing remitting multiple sclerosis (RR-MS) patients and to investigate possible clinical correlates.

Materials and methods: In all, 54 patients and 24 healthy subjects (HS) underwent multimodal magnetic resonance imaging (MRI), including diffusion tensor imaging (DTI), three-dimensional-T1-weighted and resting state (RS) functional images; they also underwent a cognitive evaluation, that is, attention and information processing speed, by means of the Paced Auditory Serial Addition Test (PASAT). Patients were also scored according to Expanded Disability Status Scale (EDSS). RS-MRI data were analysed using FMRIB Software Library (FSL) tools, with the seed-based method to identify dentate FC.

Results: When compared with HS, patients exhibited brain atrophy and widespread DTI abnormalities, as well as greater FC between the dentate nucleus and cortical areas, particularly in the frontal and parietal lobes. Within these areas, FC in patients correlated inversely with clinical impairment. Finally, FC correlated inversely with lesion load and microstructural brain damage.

Conclusion: Our findings indicate that dentate FC at rest is altered in MS patients. Whether these functional changes are induced by the disease and play a compensatory role remains to be established.

Keywords: Multiple sclerosis, functional MRI, resting state, cerebellum, plasticity, dentate nucleus

Date received: 14 January 2016; revised: 8 June 2016; accepted: 8 June 2016

\section{Introduction}

Multiple sclerosis (MS) may affect cerebellar structures by causing white matter (WM) and grey matter (GM) lesions ${ }^{1}$ as well as microstructural WM damage $^{2}$ and atrophy. ${ }^{3}$ Since the cerebellum is connected with most cortical areas, that is, sensorimotor, prefrontal, fronto-parietal and temporo-occipital cortices, ${ }^{4-6}$ any pathology affecting this region is likely to contribute to a wide range of symptoms, including motor, coordination and cognitive dysfunctions. ${ }^{7}$ Indeed, the cerebellum appears to impact on the neuro-functional connections underlying cognition, as hypothesized in the theory of 'cerebellar cognitive affective syndrome', 8 according to which cerebellar damage is accompanied by cognitive deficits and behavioural changes.
The advent of resting state functional magnetic resonance imaging (RS-fMRI) has led to cerebellar functional connectivity (FC) also being investigated. ${ }^{9-12}$ In healthy subjects (HS), the cerebellum has been shown to be functionally connected with sensorimotor areas, including the motor, premotor and somatosensory cortices as well as visual and auditory regions, and with associative prefrontal and parietal cortices. ${ }^{9}$ Within the cerebellum, the dentate nucleus, the largest nucleus involved in neocerebellar functions and the main output station, is a fundamental relay in the cortico-cerebellar circuit and is involved in both motor and cognitive performance. ${ }^{13,14}$ The dentate nucleus have been demonstrated to be involved in two main networks: a motor network, involving the dorsal dentate, the anterior cerebellar
Multiple Sclerosis Journal

$1-10$

DOI: $10.1177 /$

1352458516657438

(C) The Author(s), 2016. Reprints and permissions: http://www.sagepub.co.uk/ journalsPermissions.nav
Correspondence to: E Sbardella

Department of Neurology and Psychiatry, Sapienza University of Rome, Viale dell'Università 30, 00185 Rome, Italy. emilia.sbardella@ uniroma1.it

Emilia Sbardella Neeraj Upadhyay Francesca Tona Luca Prosperini Laura De Giglio Nikolaos Petsas Carlo Pozzilli Department of Neurology and Psychiatry, Sapienza University of Rome, Rome, Italy

Patrizia Pantano Department of Neurology and Psychiatry, Sapienza University of Rome, Rome, Italy/IRCSS Neuromed, Pozzilli (Isernia), Italy 
regions and the pre-central gyrus, and a cognitive network, involving the ventral dentate, the crus I and the prefrontal cortex. ${ }^{13}$

Although RS-fMRI is acknowledged to be a useful means of analysing FC, ${ }^{15}$ few studies on MS have used this technique to investigate the cerebellar network at rest. ${ }^{10-12}$ Moreover, only one of these studies applied a seed analysis to investigate dentate FC in a paediatric cohort of MS patients. ${ }^{11}$ The aim of our study was to assess dentate FC changes in a population of adult relapsing remitting multiple sclerosis (RR-MS) patients in order to investigate dentate RS connectivity abnormalities induced by this disease and to assess their relationship with brain structural integrity and the severity of clinical impairment.

\section{Materials and methods}

\section{Participants}

We prospectively recruited 54 patients, aged from 18 to 50 years, with a diagnosis of RR-MS according to the revised McDonald criteria ${ }^{16}$ and with an Expanded Disability Status Scale (EDSS) ${ }^{17}$ score of up to 5.0. The exclusion criteria were as follows: concomitant serious systemic diseases, that is, cardiological, pulmonary, oncological or infective pathologies, a relapse or new medication over the previous 6 months, contraindications to MRI, presence of one or more gadoliniumenhancing lesions at the baseline MRI, cognitive impairment as detected by a Mini-Mental State Examination score of less than 24. Patients underwent MRI and a clinical evaluation, including the EDSS and the Paced Auditory Serial Addition Test (PASAT) at 3 seconds. The EDSS, which is the most common method of quantifying disability in MS people, evaluates eight different functional systems (pyramidal, cerebellar, brainstem, sensory, bowel/bladder, visual, cerebral, other); the higher the EDSS score, the worse the clinical status. The PASAT is an arithmetical test in which the number that has just been heard and the number heard before have to be added; it involves attention, working memory and information processing speed, ${ }^{18}$ and is considered in this study as an index of cognitive performance. The higher the PASAT score, the better the performance; the PASAT $z$-scores were calculated according to a normative population. ${ }^{18}$ None of the patients had previously performed the PASAT.

In all, 24 matched HS, with no relevant medical disorder, constituted the control group; the HS also underwent MRI and the PASAT. The Local Ethics Committee approved the study and written informed consent was obtained from all the participants.

\section{MRI acquisition}

Imaging was performed with a Siemens Magnetom Verio 3 Tesla scanner. Slice orientation parallel to the sub-callosal line was assured by acquiring a multiplanar T1-weighted (T1-w) localizer at the beginning of each MRI acquisition.

The multimodal MRI study included the following:

1. RS-fMRI. Blood oxygen level dependent (BOLD) single-shot echo-planar images repetition time $(\mathrm{TR})=3000 \mathrm{~ms}$, echo time $(\mathrm{TE})=30 \mathrm{~ms}$, flip angle $=89^{\circ}$, matrix $=64 \times 64$, field of view $(F O V)=192 \mathrm{~mm}, 50$ contiguous axial slices, $3 \mathrm{~mm}$ thick, 140 volumes, acquisition time $=7$ minutes 11 seconds;

2. Diffusion tensor imaging (DTI). Single-shot echo-planar spin-echo images, $\mathrm{TR}=12,200 \mathrm{~ms}$, $\mathrm{TE}=94 \mathrm{~ms}$, matrix $=96 \times 96, \quad \mathrm{FOV}=192 \mathrm{~mm}$, 72 contiguous axial slices, $2 \mathrm{~mm}$ thick, $b=0$ and $1000 \mathrm{~s} / \mathrm{mm}^{2}, 30$ gradient directions;

3. T1 volumetric imaging. Three-dimensional (3D) T1-w (T13D) magnetization-prepared rapid acquisition gradient echo (MPRAGE) sequence, $\mathrm{TR}=1900 \mathrm{~ms}, \mathrm{TE}=2.93 \mathrm{~ms}$, flip angle $=9^{\circ}$, matrix $=256 \times 256, \mathrm{FOV}=260 \mathrm{~mm}$, 176 contiguous sagittal slices, $1 \mathrm{~mm}$ thick;

4. Dual turbo spin-echo, proton density and T2-w images. Axial, $\mathrm{TR}=3320 \mathrm{~ms}, \mathrm{TE}=10 / 103 \mathrm{~ms}$, $384 \times 384$ matrix, FOV $=220 \mathrm{~mm}, 25$ axial slices, $4 \mathrm{~mm}$ thick, 30\% gap;

5. T1-w spin-echo images after administration of gadolinium-based contrast agent, acquired only in patients. $\mathrm{TR}=550 \mathrm{~ms}, \mathrm{TE}=9.8 \mathrm{~ms}$, matrix $=320 \times 320, \quad F O V=240 \mathrm{~mm}, 25$ axial slices, $4 \mathrm{~mm}$ thick, 30\% gap.

The scans covered the whole brain, including the cerebellum, in all the subjects.

\section{Image processing and data analysis}

All image analyses were carried out using FMRIB Software Library (FSL) tools (the FMRIB Software Library, http://www.fmrib.ox.ac.uk/fsl), version 5.0.7.

\section{RS-fMRI analysis}

Pre-processing. RS-fMRI data were processed using a seed-based analysis. Single-subject preprocessing was performed using FMRI Expert Analysis Tool (FEAT). Pre-processing included (a) motion correction, (b) brain extraction, (c) spatial smoothing using a Gaussian kernel of full width at half maximum (FWHM) $6 \mathrm{~mm}$ and (d) high-pass 
temporal filtering using a cut-off of 150 seconds $(0.007 \mathrm{~Hz})$. Functional data were aligned to structural images (within-subject), initially using linear registration and then optimized using the boundary-based registration approach.

Structural images were transformed to standard space using a FMRIB's Non-linear Registration Tool (FNIRT), and the resulting warp fields applied to the functional statistical summary images. Two spherical $4 \mathrm{~mm}^{3}$ regions of interest (ROIs) were created using the standardized coordinates within the left and right dentate nucleus (left: $x=-17, y=-58, z=-35$; right: $x=17, y=-56, z=-35)^{19}$ on the standard Montreal Neurological Institute (MNI)_2mm template included in the FSL toolbox. These ROIs were then transformed to the individual functional data space by applying the inverse non-linear deformation matrix obtained during pre-processing of the data.

Comparison and correlation of fMRI analyses. ROIs of both dentate nuclei for each subject were used for the seed analysis to define the reference time course. A correlation analysis between each reference time course and the signal time series in each voxel within the acquired whole-brain image set was then performed. A correct overlap of seed regions on RSfMRI data was ensured by visual inspection in all the subjects enrolled. Seeds of cerebrospinal fluid (CSF) and WM were also individually defined in the lateral ventricles and in the centrum ovale on echo-planar images; their courses were then used to remove nonneural contributions to the BOLD signal and enhance specificity. The $z$-score $\mathrm{FC}$ maps for each subject were then generated by displaying all those voxels whose signal time series were significantly correlated with the seed region $(p \leqslant 0.05)$.

The within-group analysis was initially conducted using a one-sample analysis (cluster level, $p \leqslant 0.05$, family-wise error (FWE) corrected) for the patient and control groups separately to obtain $z$-score statistical maps of dentate FC. A between-group comparison was then performed using a two-sample $t$-test model (unpaired, cluster level, $p \leqslant 0.05$, FWE corrected). A mask including dentate $\mathrm{FC}$ voxels that differed significantly between patients and HS was then created (differential $\mathrm{FC}$ mask). The mean $z$-score within this mask was calculated for each subject and used to create scatter plots. In MS patients, we calculated the correlations of dentate FC within the whole brain with $\mathrm{T} 2$ lesion volume (T2LV) as well as the correlations of dentate FC within the differential FC mask with the EDSS and PASAT scores. For this purpose, one-sample analysis with covariates of interest was designed using a general linear model (GLM). In both two-sample and one-sample analyses, age, sex, cerebellar size and GM volume were entered as nointerest covariates. The correlation between FC and the PASAT was corrected for the EDSS, just as the correlation between FC and the EDSS was corrected for the PASAT. Motion parameters, which were entered as covariates of no interest although no significant difference between patients and HS was detected, ${ }^{20}$ yielded a mean absolute and relative displacement of 0.194 versus 0.210 and 0.032 versus 0.034 ( $p=0.43$ and $p=0.22$, respectively). The anatomical localization of significant clusters was established according to the Harvard-Oxford Structural Atlas, the FSL Juelich Histologic Atlas.

Tract-based Spatial Statistics and DTI parameters. 'Eddy current' correction was used to pre-process raw DTI images to correct for distortions due to the gradient directions applied. Subsequently, DTIFit was used to fit a diffusion tensor model at each voxel and generate individual fractional anisotropy (FA) maps. FA maps were fed into the Tract-Based Spatial Statistics (TBSS) tool. The FA maps of all the subjects were aligned into a common space by non-linear registration and averaged to obtain a mean FA skeleton. Finally, each subject's aligned FA data were projected onto this skeleton. A GLM was applied to assess differences in FA between patients and HS and to identify any correlation between FA and both the PASAT and EDSS scores in patients. An analysis was also performed to assess the relationship between FA in the whole brain, as well as in the superior cerebellar peduncle (SCP) and middle cerebellar peduncle (MCP), and the mean single-subject FC $z$-score obtained from the differential FC mask. Analyses were adjusted for the subjects' sex, age, brain volume and T2LV (whole as well as supratentorial/subtentorial separately). A GLM was applied using permutation-based non-parametric testing (5000 permutations, cluster level, $p \leqslant 0.05$, FWE corrected). Significant WM tracts were identified by means of the FSL WM Atlas.

Lesion volume. T2LV was calculated on PD-w images using a semi-automated technique with the Jim 5.0 software (Xinapse System, Leicester, UK). Using this technique, the operator can detect lesions, whose areas are automatically drawn and volumes calculated by the program. Supratentorial and subtentorial (brainstem, cerebellum and cerebellar peduncles) T2LV were also calculated separately. Lesion masks were created, but lesion filling was not applied.

Volumetric assessment. GM and WM volumes were obtained using SienaX, which is part of FSL, after 
Table 1. Demographic and clinical characteristics in patients and healthy subjects.

\begin{tabular}{lccc}
\hline & Patients $(n=54)$ & Healthy subject $(n=24)$ & $p$ \\
\hline Age (years) & $38.55 \pm 9.46$ & $36.32 \pm 6.81$ & 0.32 \\
Sex, f/m $(n)$ & $35 / 19$ & $14 / 10$ & 0.62 \\
EDSS (median, range) & $2.5(1-5)$ & na & na \\
Disease duration $($ years $)$ & $11.45 \pm 6.48$ & na & na \\
Cerebellar area $\left(\mathrm{cm}^{2}\right)$ & $10.36 \pm 1.52$ & $11.99 \pm 1.02$ & $<\mathbf{0 . 0 0 0 1}$ \\
Supratentorial T2LV $\left(\mathrm{cm}^{3}\right)$ & $8.71 \pm 9.61$ & na & na \\
Subtentorial T2LV $\left(\mathrm{cm}^{3}\right)$ & $0.41 \pm 0.37$ & $764.21 \pm 377.07$ & na \\
NWM volume $\left(\mathrm{cm}^{3}\right)$ & $704.59 \pm 77.78$ & $810.92 \pm 451.08$ & $\mathbf{0 . 0 0 1}$ \\
NGM volume $\left(\mathrm{cm}^{3}\right)$ & $745.40 \pm 80.73$ & $1575.14 \pm 74.36$ & $<\mathbf{0 . 0 0 0 1}$ \\
NBV $\left(\mathrm{cm}{ }^{3}\right)$ & $1452.12 \pm 153.84$ & $0.535 \pm 0.609$ & $<\mathbf{0 . 0 0 0 1}$ \\
PASAT $(z$-score) & $-0.713 \pm 1.128$ & $<\mathbf{0 . 0 0 0 1}$ \\
\hline EDSS: Expanded Disability Status Scale; T2LV: T2 lesion volume; NWM: normalized white matter; NGM: normalized grey matter; \\
NBV: normalized brain volume; na: not applicable; PASAT: Paced Auditory Serial Addition Test. \\
All values are expressed as mean \pm SD, except otherwise specified. The significant differences are shown in bold.
\end{tabular}

removing the individual lesion masks. Once the nonbrain tissue had been stripped, the brain and skull images were used to estimate the scaling between the subject's image and standard space. Following segmentation, brain tissue volumes were calculated and multiplied by the estimated scaling factor to reduce head-size-related variability between subjects and obtain normalized volumes of GM, WM and global brain volume.

As a surrogate of cerebellar volume, we calculated the cerebellar area in all the subjects on 3DT1 sequences. ${ }^{21}$ Briefly, on the midline sagittal slice, in which the aqueduct of Sylvius was more clearly visualized, an ROI was automatically created to outline the boundary between the cerebellum and CSF in the posterior fossa using Jim software.

\section{Statistical analysis}

Statistical analysis was carried out using SPSS software, version 16.0 (SPSS, Chicago, IL, USA). All values are reported as mean \pm standard deviation (SD) or median and range, as appropriate. Demographic, behavioural and radiological differences between groups were tested using the $t$ test and the exact Fisher's test for continuous and categorical variables, respectively. Spearman's analysis was used for correlations. The statistical threshold was set at $p \leqslant 0.05$.

\section{Results}

\section{Participants}

The demographic, clinical and radiological characteristics of the $54 \mathrm{MS}$ patients and $24 \mathrm{HS}$ are shown in
Table 1. WM and GM volumes, cerebellar size as well as cognitive performance at the PASAT were lower in patients than in HS.

No correlation emerged between the PASAT and EDSS scores in patients $(p=0.31)$. When we investigated the relationship between the structural data (cerebellar size, WM and GM volume, supra- and subtentorial T2LV) and the EDSS and PASAT scores, T2LV within the whole brain and T2LV at the supratentorial level was found to correlate directly with the EDSS scores ( $p=0.01, p=0.009$, respectively).

\section{Dentate FC and correlations with T2LV and clinical data}

HS displayed widespread dentate FC with cortical areas, located in the frontal, temporal, parietal and occipital lobe, and with deep GM, including the thalamus and striatum, bilaterally (Figure 1(a)). Dentate FC with several cortical areas was greater in patients than in HS, particularly in the frontal (superior, middle and inferior frontal gyri, supplementary motor area (SMA) and pre-central gyrus, bilaterally) and parietal (supramarginal gyrus, precuneus, superior parietal lobule and post-central gyrus, bilaterally) cortices (Figure 1(b), Table 2).

Dentate FC with the cerebellum, right thalamus, frontal and parieto-occipital cortices correlated inversely with T2LV (Figure 2), that is, the higher the FC, the lower the lesion burden.

Dentate FC with superior and middle frontal, pre- and post-central gyri in the right and SMA 


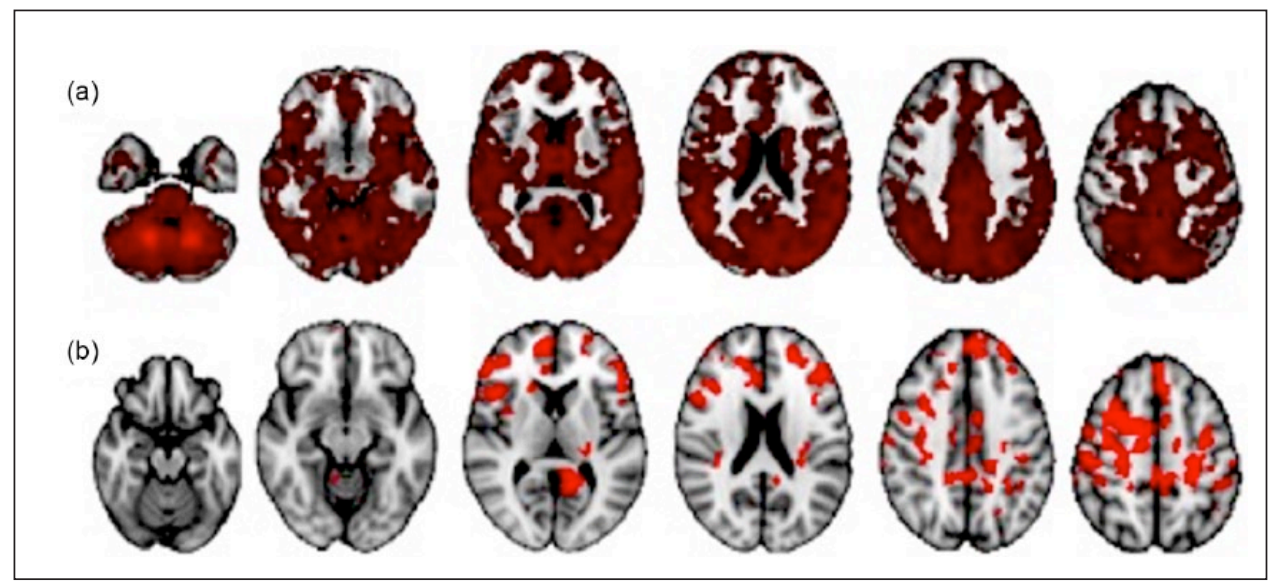

Figure 1. (a) Dentate functional connectivity in healthy subjects (red). (b) Areas (red) of higher dentate functional connectivity in patients than in healthy subjects, above all in the superior, middle and inferior frontal gyri, the supplementary motor area and the pre-central gyrus bilaterally, in the supramarginal gyrus, precuneus, superior parietal lobule and post-central gyrus bilaterally.

Differences were calculated using the unpaired $t$ test; results are cluster level, family-wise error corrected, $p<0.05$. Areas were identified using the Harvard Probabilistic Atlas and are overlapped on an MNI_2mm image.

Table 2. Areas of differences in dentate functional connectivity between patients and healthy subjects.

\begin{tabular}{|c|c|c|c|c|c|}
\hline Brain area & Voxels & $z$ value & $x$ & $y$ & $z$ \\
\hline $\mathrm{R}$ middle frontal gyrus & 534 & 3.78 & 30 & 8 & 54 \\
\hline L supramarginal gyrus & 296 & 4.35 & -34 & -40 & 32 \\
\hline $\mathrm{R}$ inferior frontal gyrus & 234 & 4.02 & 50 & 16 & 12 \\
\hline L posterior cingulate cortex & 279 & 3.91 & -14 & -50 & 12 \\
\hline L superior frontal gyrus & 235 & 3.67 & -4 & 24 & 54 \\
\hline L pre-central gyrus & 174 & 3.7 & -28 & -24 & 54 \\
\hline R pre-central gyrus & 250 & 3.73 & 30 & 6 & 30 \\
\hline L inferior frontal gyrus & 99 & 3.54 & -48 & 28 & 4 \\
\hline $\mathrm{R}$ posterior cingulate gyrus & 96 & 3.84 & 22 & -40 & 38 \\
\hline $\mathrm{L}$ middle frontal gyrus & 95 & 3.3 & -48 & 28 & 28 \\
\hline R postcentral gyrus & 74 & 3.76 & 48 & -22 & 52 \\
\hline $\mathrm{R}$ medial frontal gyrus & 72 & 3.25 & 12 & 52 & 8 \\
\hline L postcentral gyrus & 59 & 3.36 & -22 & -34 & 50 \\
\hline L precuneus & 63 & 3.3 & -12 & 46 & 38 \\
\hline $\mathrm{R}$ paracingulate gyrus & 46 & 3.17 & 16 & 20 & 34 \\
\hline $\mathrm{R}$ anterior cingulate gyrus & 33 & 3.49 & 8 & 30 & 16 \\
\hline R supplementary motor area & 23 & 3.05 & 12 & -14 & 52 \\
\hline R caudate & 19 & 3.32 & 20 & 22 & 8 \\
\hline L supplementary motor area & 14 & 3.08 & -12 & -14 & 54 \\
\hline R superior frontal gyrus & 14 & 2.75 & 16 & 40 & 32 \\
\hline L superior parietal lobule & 12 & 2.96 & -42 & -38 & 56 \\
\hline $\mathrm{L}$ anterior cingulate cortex & 10 & 2.75 & -6 & -4 & 44 \\
\hline $\mathrm{R}$ cerebellum anterior lobe & 10 & 2.43 & 8 & -50 & 12 \\
\hline
\end{tabular}

bilaterally correlated inversely with the EDSS scores (Figure 3(a)), that is, the higher the FC, the lower the clinical disability. Correction for the PASAT scores did not change the results. 


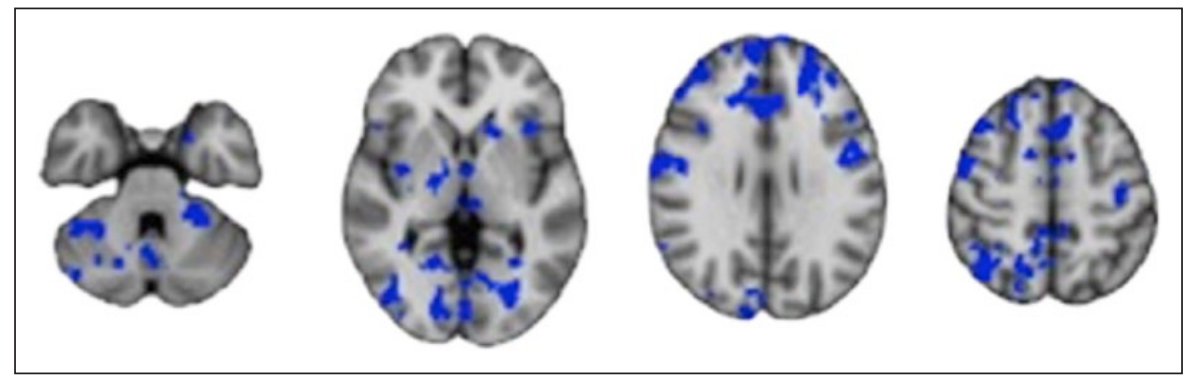

Figure 2. Areas (blue) that yielded an inverse correlation between dentate functional connectivity and T2-lesion volume in patients.

Results are cluster level, family-wise error corrected, $p<0.05$. Areas were identified using the Harvard Probabilistic Atlas and are overlapped on an MNI_2mm image.

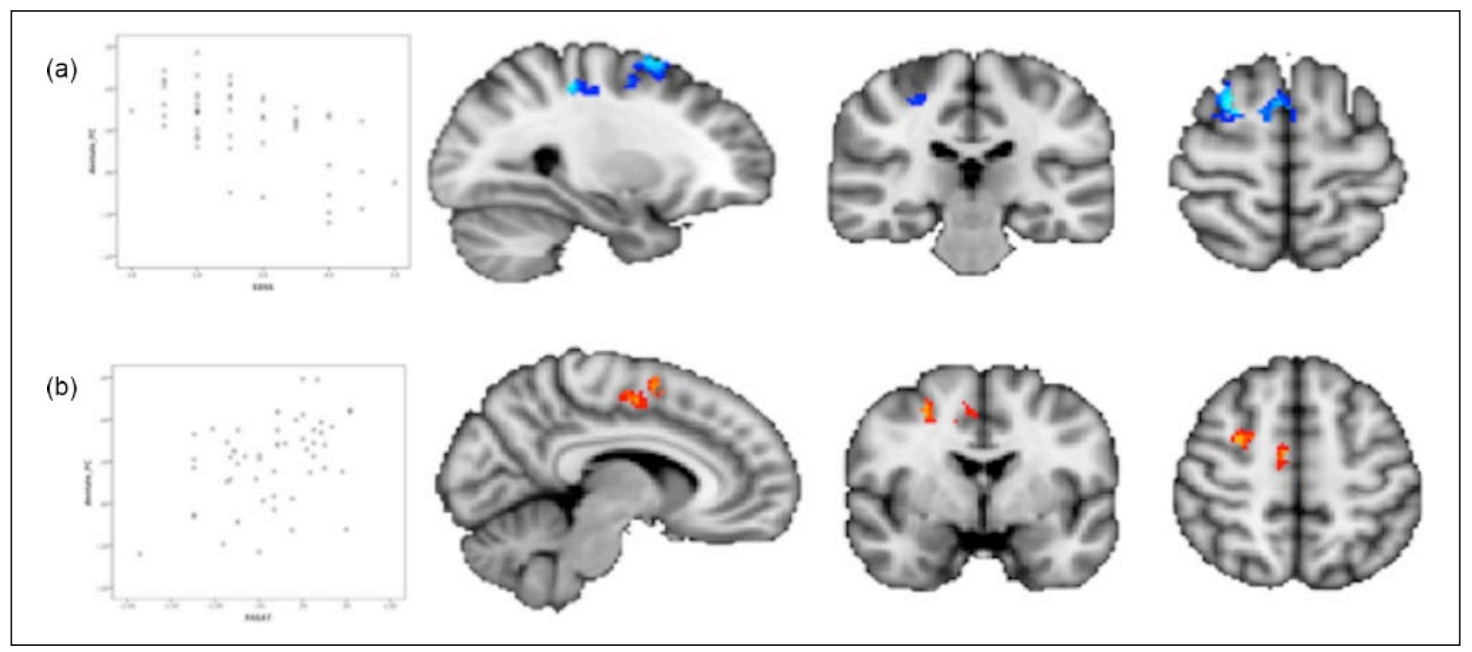

Figure 3. Patients. (a). Areas that yielded an inverse (blue) correlation between dentate functional connectivity and EDSS, within the superior and middle frontal and pre- and post-central gyri on the right and SMA bilaterally. Scatter plot showing the inverse correlation $(p<0.001, \rho=-0.52$ ). (b) Areas that yielded a direct (red) correlation between dentate functional connectivity and the PASAT score within the superior and middle frontal gyrus, cingulate gyrus and SMA on the right. Scatter plot showing the direct correlation $(p=0.001, \rho=0.43)$.

Results are cluster level, family-wise error corrected, $p<0.05$. For the scatter plots, the $\rho$ value was obtained by means of Spearman's analysis. Areas were identified using the Harvard Probabilistic Atlas and are overlapped on an MNI_2mm image.

Dentate FC with the superior, middle frontal and cingulate gyri and SMA on the right directly correlated with the PASAT scores (Figure 3(b)), that is, the higher the FC, the better the performance. Correction for the EDSS scores did not change the results.

\section{DTI and correlation with clinical data}

Patients displayed a significant reduction in FA values throughout the skeleton, involving all the main WM tracts, particularly in the corpus callosum, anterior thalamic radiations, inferior and superior longitudinal fasciculi, cerebellar peduncles and cortico-spinal tracts (Figure 4(a)).

Furthermore, FA values in patients in most of the aforementioned WM tracts correlated directly with the PASAT scores (Figure 4(b)), that is, the better preserved the microstructural WM integrity, the better the cognitive performance. No correlation was detected between FA and the EDSS.

\section{Correlation between DTI data and dentate FC}

The mean $z$-scores within the differential FC mask directly correlated with FA values in most of the WM tracts within the whole skeleton, that is, in the corticospinal tracts bilaterally, superior longitudinal and fronto-occipital fasciculi, anterior and posterior corpus callosum, and cerebellar peduncles (Figure 5(a)), as well as with FA values within the SCP and MCP bilaterally (Figure 5(b)). These results indicate that the more preserved the microstructural WM integrity, the higher the FC. 


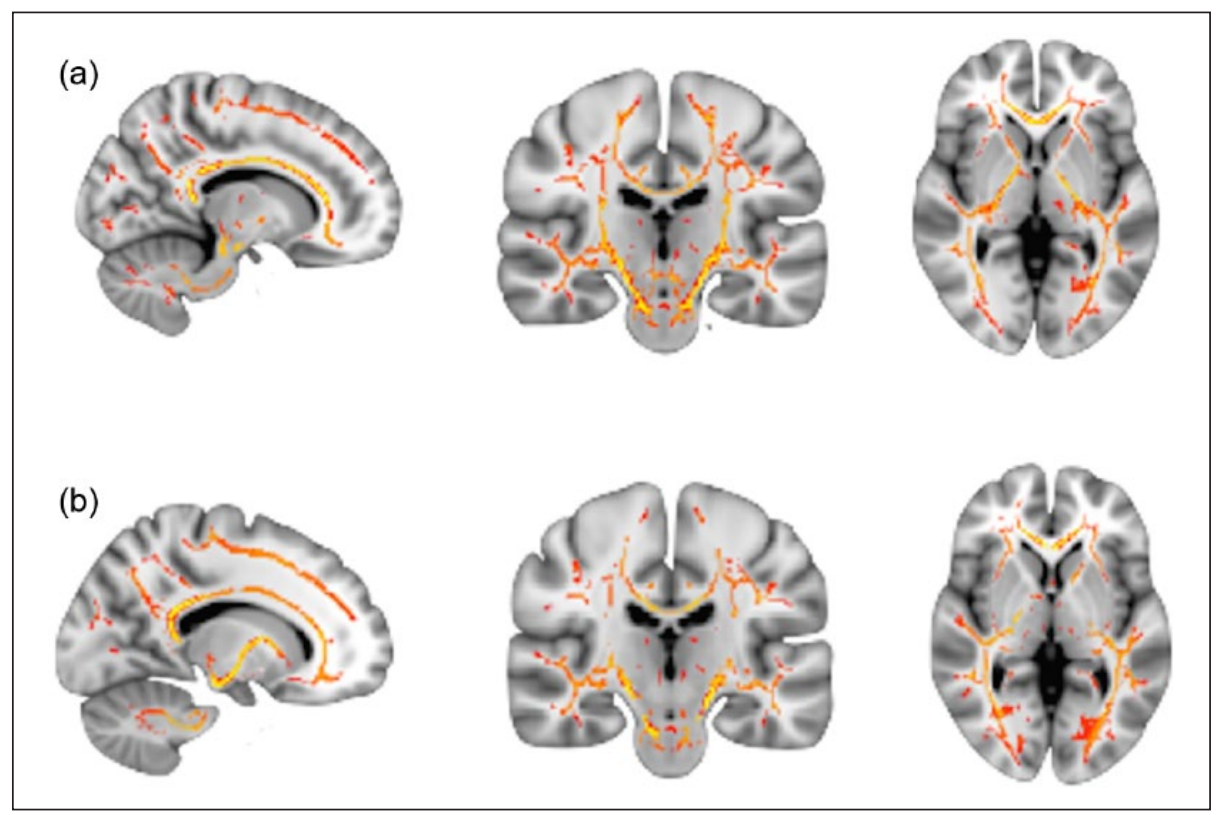

Figure 4. (a). Areas (red-yellow) of reduced fractional anisotropy in patients compared with healthy subjects throughout the skeleton. (b) Areas (red-yellow) yielding a direct correlation between fractional anisotropy values and the PASAT score in patients within the semi-ovale centre, capsule, thalamic radiation, cerebellum and superior longitudinal fasciculus bilaterally.

Differences were calculated using the unpaired $t$ test; results are cluster level, family-wise error corrected, $p<0.05$. Areas were identified using the Harvard Probabilistic Atlas and are overlapped on an MNI_2mm image.

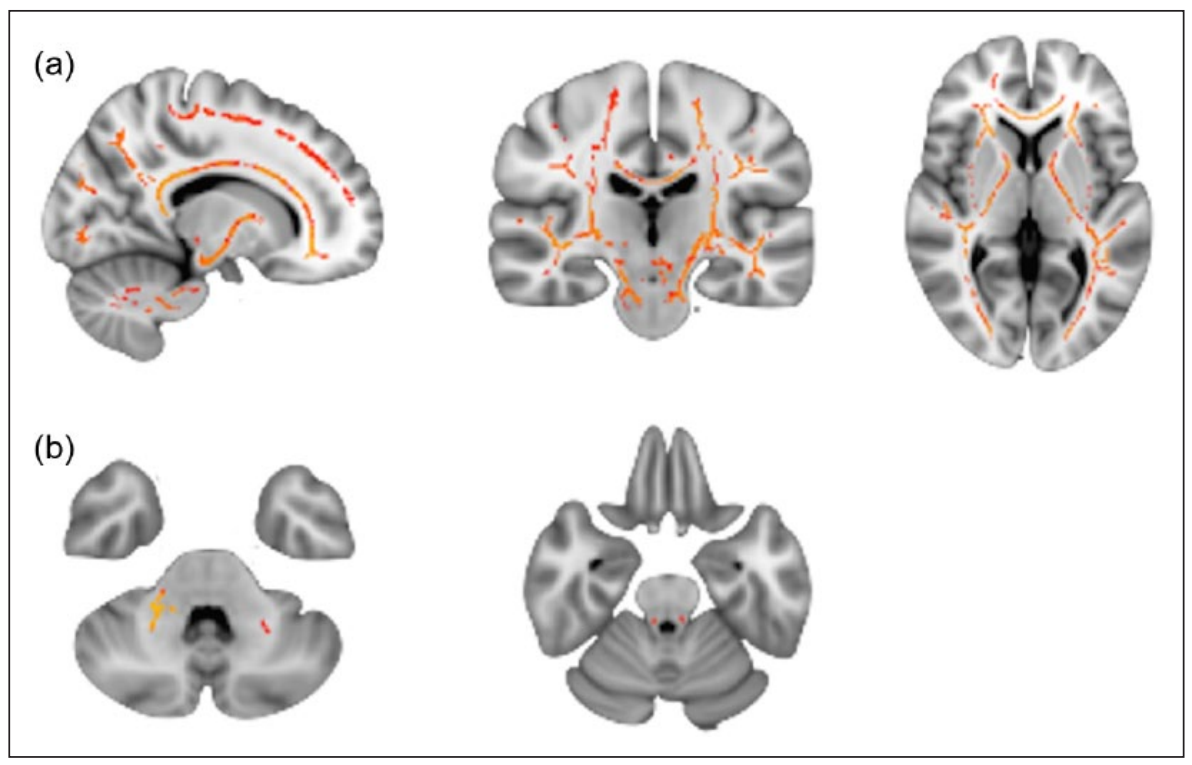

Figure 5. Direct correlations (red-yellow) between FC values and: (a) fractional anisotropy in the whole brain of patients; (b) fractional anisotropy in the middle (left) and superior (right) cerebellar peduncles of patients.

Results are cluster level, family-wise error corrected, $p<0.05$. Areas were identified using the Harvard Probabilistic Atlas and are overlapped on an MNI_2mm image.

\section{Discussion}

In both patients and HS, we detected significant functional connections between the dentate nucleus and both subcortical and cortical areas involved in sensorimotor control and high-level functions. Patients displayed higher dentate FC with frontal areas than HS, particularly with the frontal and precentral gyri and SMA, as well as and with the parietal 
cortex. Increased dentate FC was associated with lower structural tissue damage, as demonstrated by T2LV and FA values, and lower severity of clinical impairment, in terms of both motor and cognitive involvement as measured by the EDSS and PASAT scores, respectively.

Our results are in keeping with previous functional and structural data from studies that reported cerebellar connections with prefrontal, motor and parietal cortices, ${ }^{5,6,9}$ areas that are widely known to contribute to motor and higher cognitive function. ${ }^{6,22}$

The clinical involvement in patients in this study was relatively mild, as demonstrated by the low EDSS mean value and PASAT scores that were fairly well preserved despite widespread WM structural damage involving even the long connecting fibres, such as the cortico-cerebellar tracts, which are usually involved in MS. ${ }^{23}$ The fact that FC was higher in patients than in HS might be interpreted as an adaptive plastic change aimed at compensating for clinical impairment. Indeed, a previously reported increase in $\mathrm{FC}$ in the early stages of the disease in the absence of clinical involvement was considered as an attempt to recruit reserve capacity aimed at compensating for damage. ${ }^{24}$ However, a recent cognitive study that investigated the dentate network in MS children reported areas of both increased and decreased dentate FC in patients in comparison with $\mathrm{HS},{ }^{11}$ thus challenging the compensative hypothesis. Nevertheless, the impaired myelin maturation of the cerebellar circuits in childhood might partially explain differences between MS children and our adult population.

The inverse correlation of dentate FC with both structural damage, including the principal cerebellar connecting input and output fibres within SCP and MCP, and with clinical impairment further supports the hypothesis of increased FC as an adaptive plastic mechanism, as has been suggested in previous studies. ${ }^{24-27}$ Greater FC in other networks, that is, the default, frontoparietal and sensorimotor networks, in patients with normal cognitive performance and lower structural damage than in patients with cognitive impairment and greater structural damage, has been reported in previous studies, ${ }^{24-27}$ in which it was interpreted as a compensatory mechanism.

However, owing to the cross-sectional design of our study, it is impossible to determine with certainty whether preserved clinical abilities are due to increased dentate FC, to lesser structural tissue damage, or both. The finding of a direct correlation between T2LV and EDSS, as well as between FA values and cognitive performance as measured by the PASAT, supports the role of structural integrity in preserving clinical performance. ${ }^{28,29}$ In the light of these results, the hypothesis of increased FC as a compensative adaptation mechanism would be weakened, while its role as an epiphenomenon, or even as a maladaptive response without any clinical benefit, would be strengthened. ${ }^{30-32}$

Finally, the correlation of dentate FC with the EDSS, as well as the correlation of both dentate FC and cerebellar peduncle structural integrity with the PASAT, confirms the involvement of cerebellar-cortical connections in motor and non-motor abilities.

Our study has certain limitations. Despite the crucial role played by the dentate, it is possible that other parts of the cerebellum not considered by us are involved in clinical functioning and may have affected the results. We investigated the whole dentate nucleus, without distinguishing between the ventral and dorsal parts, which are related to motor and cognitive performance, respectively. ${ }^{13}$ However, nuclear segmentation in patients in whom there is a pathological involvement of the nucleus ${ }^{33,34}$ might lead to analysis biases. Although we investigated cognition by means of the PASAT alone, it is worth bearing in mind that this test has proved reliable in detecting MS-induced cognitive failure in the clinical setting. ${ }^{35}$ The fact that we used the EDSS score without considering its subsystems might also have affected some of our results. Lastly, we did not perform lesion filling although we did remove the lesion masks from the volumetric assessment to avoid any misleading registration and segmentation.

In conclusion, our results show increased dentate FC with motor and non-motor cortical areas in $\mathrm{MS}$ patients. Whether the increased FC is an adaptive or maladaptive effect consequent to structural WM damage may only be established by planning further studies based on a longitudinal design.

\section{Acknowledgements}

The authors thank all the patients who participated in the study.

\section{Declaration of Conflicting Interests}

The author(s) declared no potential conflicts of interest with respect to the research, authorship, and/or publication of this article.

\section{Funding}

The author(s) received no financial support for the research, authorship, and/or publication of this article. 


\section{References}

1. Kutzelnigg A, Faber-Rod JC, Bauer J, et al. Widespread demyelination in the cerebellar cortex in multiple sclerosis. Brain Pathol 2007; 17(1): 38-44.

2. Deppe M, Tabelow K, Krämer J, et al. Evidence for early, non-lesional cerebellar damage in patients with multiple sclerosis: DTI measures correlate with disability, atrophy, and disease duration. Mult Scler 2015; 22(1): 73-84.

3. Calabrese M, Mattisi I, Rinaldi F, et al. Magnetic resonance evidence of cerebellar cortical pathology in multiple sclerosis. J Neurol Neurosurg Psychiatry 2010; 81(4): 401-404.

4. Habas $\mathrm{C}$ and Cabanis EA. Anatomical parcellation of the brainstem and cerebellar white matter: A preliminary probabilistic tractography study at 3T. Neuroradiology 2007; 49(10): 849-863.

5. Jissendi P, Baudry S and Balériaux D. Diffusion tensor imaging (DTI) and tractography of the cerebellar projections to prefrontal and posterior parietal cortices: A study at 3T. J Neuroradiol 2008; 35(1): 42-50.

6. Palesi F, Tournier J-D, Calamante F, et al. Contralateral cerebello-thalamo-cortical pathways with prominent involvement of associative areas in humans in vivo. Brain Struct Funct 2015; 220(6): 3369-3384.

7. Timmann D, Drepper J, Frings M, et al. The human cerebellum contributes to motor, emotional and cognitive associative learning. A review. Cortex 2010; 46(7): 845-857.

8. Schmahmann JD and Sherman JC. The cerebellar cognitive affective syndrome. Brain 1998; 121(Pt 4): $561-579$.

9. O'Reilly JX, Beckmann CF, Tomassini V, et al. Distinct and overlapping functional zones in the cerebellum defined by resting state functional connectivity. Cereb Cortex 2010; 20(4): 953-965.

10. Dogonowski A-M, Andersen KW, Madsen KH, et al. Multiple sclerosis impairs regional functional connectivity in the cerebellum. Neuroimage Clin 2014; 4: 130-138.

11. Cirillo S, Rocca MA, Ghezzi A, et al. Abnormal cerebellar functional MRI connectivity in patients with paediatric multiple sclerosis. Mult Scler 2015; 22(3): 292-301.

12. Romascano D, Meskaldji D-E, Bonnier G, et al. Multicontrast connectometry: A new tool to assess cerebellum alterations in early relapsing-remitting multiple sclerosis. Hum Brain Mapp 2015; 36(4): 1609-1619.

13. Bernard JA, Peltier SJ, Benson BL, et al. Dissociable functional networks of the human dentate nucleus. Cereb Cortex 2014; 24(8): 2151-2159.
14. Habas C. Functional imaging of the deep cerebellar nuclei: A review. Cerebellum 2010; 9(1): 22-28.

15. Beckmann CF, DeLuca M, Devlin JT, et al. Investigations into resting-state connectivity using independent component analysis. Philos Trans $R$ Soc Lond B Biol Sci 2005; 360(1457): 1001-1013.

16. Polman $\mathrm{CH}$, Reingold $\mathrm{SC}$, Banwell B, et al. Diagnostic criteria for multiple sclerosis: 2010 revisions to the McDonald criteria. Ann Neurol 2011; 69(2): 292-302.

17. Kurtzke JF. Rating neurologic impairment in multiple sclerosis: An expanded disability status scale (EDSS). Neurology 1983; 33(11): 1444-1452.

18. Uitdehaag BMJ, Adèr HJ, Roosma TJA, et al. Multiple sclerosis functional composite: Impact of reference population and interpretation of changes. Mult Scler 2002; 8(5): 366-371.

19. Dimitrova A, Zeljko D, Schwarze F, et al. Probabilistic 3D MRI atlas of the human cerebellar dentate/interposed nuclei. Neuroimage 2006; 30(1): $12-25$.

20. Power JD, Barnes KA, Snyder AZ, et al. Spurious but systematic correlations in functional connectivity MRI networks arise from subject motion. Neuroimage 2012; 59(3): 2142-2154.

21. Prosperini L, Petsas N, Raz E, et al. Balance deficit with opened or closed eyes reveals involvement of different structures of the central nervous system in multiple sclerosis. Mult Scler 2014; 20(1): 81-90.

22. Sarica A, Cerasa A and Quattrone A. The neurocognitive profile of the cerebellum in multiple sclerosis. Int J Mol Sci 2015; 16(6): 12185-12198.

23. Anderson VM, Wheeler-Kingshott CAM, Abdel-Aziz $\mathrm{K}$, et al. A comprehensive assessment of cerebellar damage in multiple sclerosis using diffusion tractography and volumetric analysis. Mult Scler 2011; 17(9): 1079-1087.

24. Roosendaal SD, Schoonheim MM, Hulst HE, et al. Resting state networks change in clinically isolated syndrome. Brain 2010; 133(Pt 6): 1612-1621.

25. Rocca MA, Valsasina P, Absinta M, et al. Defaultmode network dysfunction and cognitive impairment in progressive MS. Neurology 2010; 74(16): $1252-1259$.

26. Bonavita S, Gallo A, Sacco R, et al. Distributed changes in default-mode resting-state connectivity in multiple sclerosis. Mult Scler 2011; 17(4): 411-422.

27. Cruz-Gómez AJ, Ventura-Campos N, Belenguer A, et al. The link between resting-state functional connectivity and cognition in MS patients. Mult Scler 2013; 20(3): 338-348. 
Visit SAGE journals online http://msj.sagepub.com

(SAGE journals
28. Sbardella E, Tona F, Petsas N, et al. DTI measurements in multiple sclerosis: Evaluation of brain damage and clinical implications. Mult Scler Int 2013; 2013: 671730 .

29. Sbardella E, Petsas N, Tona F, et al. Assessing the correlation between grey and white matter damage with motor and cognitive impairment in multiple sclerosis patients. PLoS ONE 2013; 8(5): e63250.

30. Tona F, Petsas N, Sbardella E, et al. Multiple sclerosis: Altered thalamic resting-state functional connectivity and its effect on cognitive function. Radiology 2014; 271(3): 814-821.

31. Faivre A, Rico A, Zaaraoui W, et al. Assessing brain connectivity at rest is clinically relevant in early multiple sclerosis. Mult Scler 2012; 18(9): 1251-1258.

32. Sbardella E, Tona F, Petsas N, et al. Functional connectivity changes and their relationship with clinical disability and white matter integrity in patients with relapsing-remitting multiple sclerosis. Mult Scler 2015; 21(13): 1681-1692.

33. Roccatagliata L, Vuolo L, Bonzano L, et al. Multiple sclerosis: Hyperintense dentate nucleus on unenhanced T1-weighted MR images is associated with the secondary progressive subtype. Radiology 2009; 251(2): 503-510.

34. Absinta M, Rocca MA and Filippi M. Dentate nucleus T1 hyperintensity in multiple sclerosis. AJNR Am J Neuroradiol 2011; 32(6): E120-E121.

35. López-Góngora M, Querol L and Escartín A. A oneyear follow-up study of the Symbol Digit Modalities Test (SDMT) and the Paced Auditory Serial Addition Test (PASAT) in relapsing-remitting multiple sclerosis: An appraisal of comparative longitudinal sensitivity. BMC Neurol 2015; 15: 40. 